
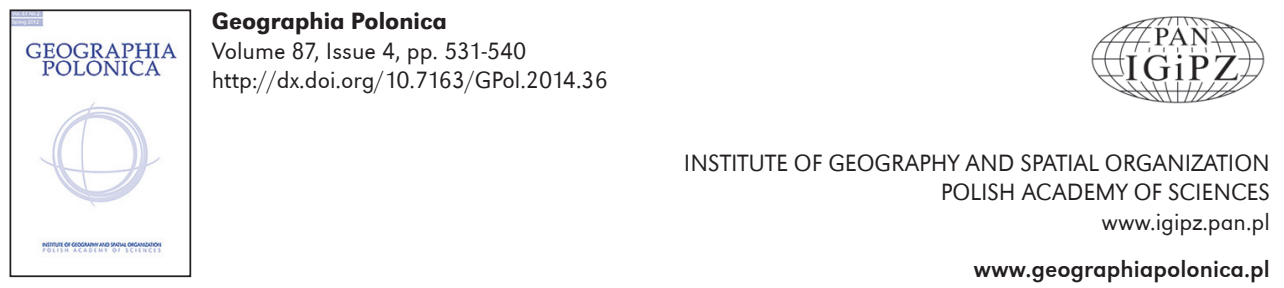

\title{
IMPACT OF MITIGATION MEASURES ON THE URBAN HEAT ISLAND PHENOMENON IN THE CITY OF MODENA, ITALY
}

\section{Stefano Marchesi - Stefano Zauli Sajani - Paolo Lauriola}

Arpa Emilia-Romagna

Regional Thematic Centre of Environment and Health

Via A. Begarelli 13, 41121 Modena: Italy

e-mails: smarchesi@arpa.emr.it•szauli@arpa.emr.it•plauriola@arpa.emr.it

\begin{abstract}
Urban planners are increasingly paying more attention to the topic of UHI. Within the framework of the EU-UHI Project, an industrial area inside the municipality of Modena was selected as a pilot-area for the Emilia-Romagna Region. In this paper we present some results obtained with RayMan, a model which estimates bioclimatic discomfort based on the human radiative budget. Some simulations of the impact of mitigation measures have been carried out, taking into account the insertion of greenery and building modifications. Both intra-diurnal and whole day effects have been compared.
\end{abstract}

\section{Keywords}

thermal discomfort $\bullet$ bioclimatic indices $\bullet$ urban planning $\bullet$ mitigation strategies

\section{Introduction}

Urban heat island (also indicated by the acronym UHI) is a microclimatic phenomenon which occurs within metropolitan areas and consists of a significant increase of urban temperature with respect to the suburbs and, in particular, to the surrounding rural areas. This difference can amount up to 1-2 degrees in the annual average, with relevant spatial and temporal variability.

For many years, UHI has been a wellknown and well-documented phenomenon and it is mainly caused by the thermal and radiative characteristics of the typical materials inside urban areas (asphalt and concrete among others), in which absorption of solar radiation prevails over reflection, and high temperatures determine a large amount of long-wave emission.

Human activities concentrated inside urban areas certainly contribute in their turn to the heating of cities, both directly (industrial activities, traffic and heating/cooling plants) and indirectly (modification of the radiative properties of the atmosphere as 
a consequence of high pollution levels associated with the aforementioned activities). Another aspect of primary importance deals with the presence of vegetation inside urban territory, acting towards a cooling of the atmosphere through latent heat.

UHI intensity is likely to be exacerbated within the next decades as a consequence of the predicted increase of mean temperature, in the framework of climate change simulation scenarios. In addition, the situation within urban areas is also expected to worsen because of the larger anthropogenic heat production associated to urban population increase. The segments of society most vulnerable to the changes will be young children and the elderly, as well as the infirm.

For all these varying reasons, UHI has recently become one of the topics which urban planners have been paying major attention to, in order to define proper mitigation measures and to promote health protection and quality of life within urban areas. For this purpose a proper modeling of the phenomenon is required to estimate the impact of the mitigation measures and to finally support local stakeholders in the development of effective strategies in the framework of urban planning.

The attention of the European Union to this problem is shown by the funding of the Project 'Development and application of mitigation and adaptation strategies and measures for counteracting the global Urban Heat Islands phenomenon (UHI)' which is coordinated by the Regional Agency for Environmental Protection in Emilia-Romagna, Italy (Arpa-ER). The Project involves 17 partners within Central Europe; in the framework of the Project, 8 pilot areas have been identified by project partners inside each of the major metropolitan areas of the Region (Budapest, Ljubljana, Modena, Padua, Prague, Stuttgart, Warsaw and Wien). The direct participation of local stakeholders guarantees the possibility of an effective impact of UHI-project objectives onto planning strategies, with a specific emphasis on the human bio-meteorological factors that are relevant for the urban planning process.
The aforementioned urban areas have been characterized in terms of the intensity of urban heat island phenomenon, making use of the available observational data (mainly meteorological). After this preliminary characterization, some pilot areas (one for each of the aforementioned cities) were selected by UHI-project partners. The main goal was to define an area where some concrete mitigation measures could be developed. In fact, a result which is foreseen in the framework of the UHI-project is the definition and the sharing of a certain number of mitigation strategies to counteract urban heat island phenomenon in an effective way from the early stages of urban area planning. In addition, the limited extension of the chosen pilot areas could also allow for the possibility of applying a modeling tool in order to evaluate the effect of the mitigation measures from a quantitative point of view.

\section{The UHI pilot area in Emilia-Romagna}

As for the municipality of Modena, local stakeholders have selected the so-called Villaggio Artigiano in the western part of the urban territory, since the area is already the subject of an urban redevelopment plan. The Villaggio Artigiano was built up at the end of 1950s and it was a pioneering experience of public and private partnership, in which the Municipality offered the area and people the know-how in order to develop small- and medium-size factories (see Fig. 1 for a general overview of the area).

At the beginning, this area was really a small village in the sense that it was almost in the countryside of Modena (which was much smaller than today); most buildings in the area were industrial warehouses, but there were also houses, shops and even a church there. Currently, the area is no longer a village, strictly speaking, since it is located in a very central position inside the urban area of Modena. In addition, some factories have discontinued their activities; nevertheless, the area maintains its distinctive 


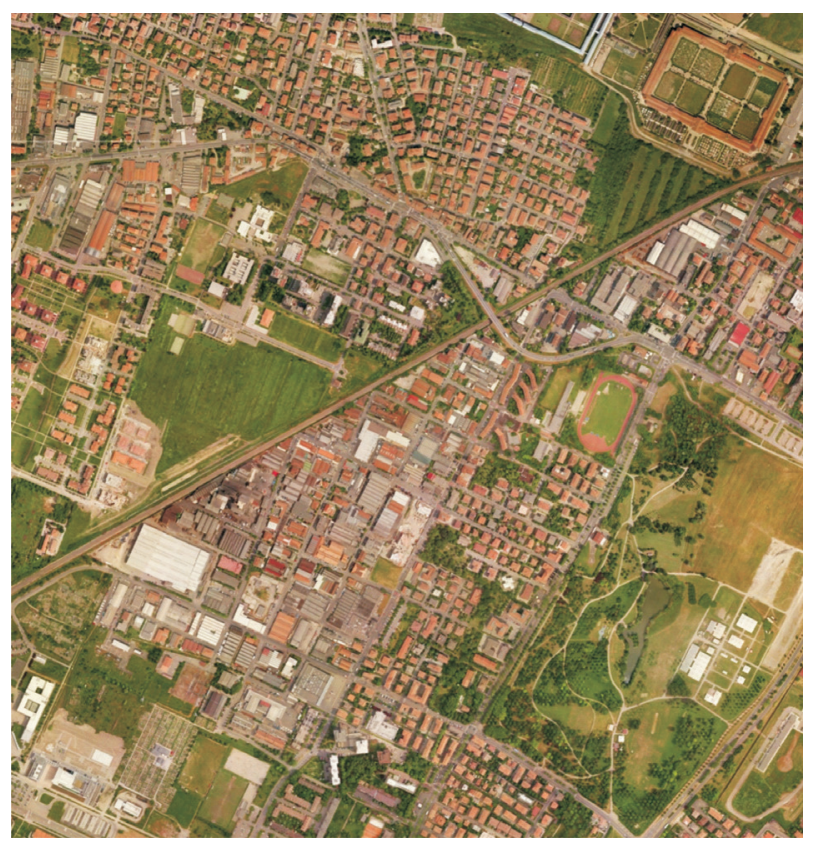

Figure 1. Aerial view of the triangle-shaped Villaggio Artigiano in Modena, in the center of the picture; buildings inside the area are clearly different with respect to the residential areas surrounding Villaggio Artigiano, both in the northern and western sides of the map

Source: Google Maps.

features and local stakeholders are mainly thinking in terms of urban regeneration rather than urban redevelopment, in order to conform the area to different requirements, whilst still maintaining the original fabric of the district.

In this paper, only a small portion inside Villaggio Artigiano has been taken into consideration (Fig. 2) with a surface area of $120 \times 120 \mathrm{~m}^{2}$ and a car-park located in the center, surrounded by 19 buildings with various streets; no green areas are present at the moment. Only 4 of the buildings are over 10-meters high (the highest reaches 13 meters); as for the remaining 15 buildings, 8 fall in the range of 8-9 meters, 3 in the range of 6-7 meters and 4 in the range of 3-5 meters: this height distribution is more typical of industrial districts rather than residential areas.

In the framework of urban regeneration, this area is particularly suitable for the introduction of relatively simple mitigation measures: in fact, there is a car park in the center of the terrain (Fig. 2), where either the insertion of trees or the setting of a grass

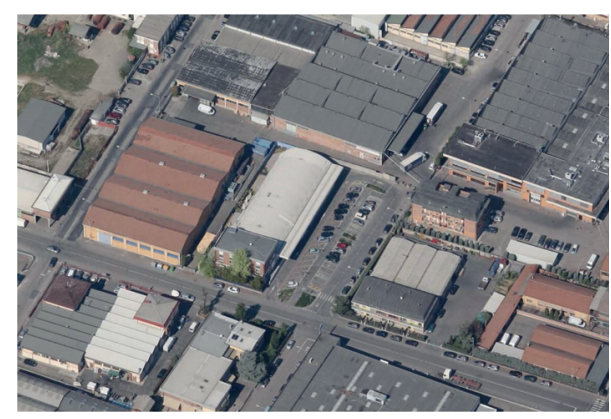

Figure 2. View of a small area inside Villaggio Artigiano, where the specific mitigation measures were applied, in particular to the car park, which is clearly visible in the center of the selected area Source: Google Maps. 
courtyard are immediate urban heat island mitigation measures in the area.

\section{The thermal indices}

At present, there is an increasing demand for bio-meteorological information, also related to the presence of the urban heat island phenomenon and its intensity, since the simple measurement of temperature and relative humidity of the atmosphere is not completely adequate for the assessment of the thermal influence of the environment on the human body. In particular, the evaluation of the thermal component of climate inside urban areas is a very challenging subject and a large number of bio-meteorological indices have been developed to date. These indices can be used in order to evaluate the impact of the various mitigation measures as will be shown later.

The parameter synthesizing the bio-meteorological influence of short- and long-wave radiation fluxes within urban areas, is the so-called mean radiant temperature, which is defined as the temperature of a spherical surface surrounding the human body giving, as a result, the same net exchange with the subject as the actual radiative environment. The mean radiant temperature can be considered as the most relevant meteorological input parameter in the context of human energy balance especially during summer.

The definition of the thermo-physiologically relevant indices starts with the setting of a heat-balance model of the human body for the assessment of thermal comfort. Several models have been defined so far since pioneering works (Büttner 1938), but the basis for all of them is the heat-balance equation which represents the heat exchange between the human body and the environment:

$$
\mathrm{M}+\mathrm{W}+\mathrm{C}+\mathrm{K}+\mathrm{E}+\mathrm{Q}+\operatorname{Res} \pm \mathrm{S}=\mathrm{O}
$$

In the equation, heat production is represented by the terms $M$ (metabolism) and W (muscular activity), while heat can be released in the environment through convection $(C)$, conduction $(K)$, evaporation $(E)$, radiation $(\mathrm{Q})$ and respiration (Res). The balance between heat production and loss must reduce term $S$ (heat content in the body) to 0 in order to maintain core body temperature within a narrow range around $37^{\circ} \mathrm{C}$.

The different models become then the basis of the calculation of universal thermal indices and then for a physiologically significant characterization of bio-meteorological conditions. Among others, common thermal indices are PET (Physiologically Equivalent Temperature; Höppe 1999) and UTCI (Universal Thermal Climate Index; Błażejczyk et al. 2013) which can be mentioned for the assessment of thermal comfort. Both indices have the same unit as temperature $\left({ }^{\circ} \mathrm{C}\right)$ and this makes them more suitable than other indices, such as the Predicted Mean Vote (PMV; Fanger 1970). In addition to having a solid physiological basis, they can be thought of as a temperature for all practical purposes, thus bridging the gap among all people involved in this issue, regardless of their scientific and technical background.

\section{The simulation model}

Several tools for urban area simulations have been defined in the framework of the UHI-project and have been applied to the pilot areas of the aforementioned metropolitan areas in Central Europe, in order to estimate the effect of some urban heat island mitigation measures from a quantitative point of view, too.

Among others, RayMan is a simulation model to calculate short- and long-wave radiation fluxes inside a complex urban environment in order to define a mean radiant temperature which a person located inside the area is exposed to. Model output is represented by the aforementioned thermal indices, defining the heat stress to which a person is subjected (Matzarakis et al. 2007, 2010).

The RayMan model is a tool which is based on the calculation of the mean radiant temperature using a simplified radiation balance applied to a person, who is exposed to direct solar radiation and to long wave 
radiation from the ground, building walls and vegetation, as well as reflected radiation from the same surfaces. The person under consideration is a 35 year old male, $1.75 \mathrm{~m}$ tall and weighing $75 \mathrm{~kg}$. Another relevant aspect is related to his activity, which is set to a working metabolism of $80 \mathrm{~W}$ (which corresponds to light activity) in addition to the basic metabolism, and to his clothing, which is set to a heat resistance of clothing equal to 0.9 clo.

The meteorological parameters which are of dominant importance for the human energy balance are air temperature, relative humidity and wind speed; all of these parameters have to be measureable at a biometeorologically significant height, which is set as $1.1 \mathrm{~m}$ above the ground (the average height of a standing person's center of gravity).

Output from the RayMan model consists of the values of a series of thermal indices derived from the human heat-balance model: these kinds of indices are proven to give a better representation of the estimate of the thermo-physiological effect of the atmospheric environment with respect to the simple bioclimatic indices used so far. In this respect, the comfort/discomfort conditions of a human being at the center of the heat balance model can be assessed using these indices.

In the present study, the RayMan model has been used to calculate the heat balance at the center of a domain located around a car park inside Villaggio Artigiano (Fig. 2). The RayMan structure makes it particularly suitable for this kind of micro-scale analysis: in other words, the model is run for the actual situation in the domain (reference run), and thermal indices are computed according to typical summer conditions (August 5th, 2012): the values for the model meteorological input (air temperature, relative humidity and wind speed) are obtained from the observations collected by the urban meteorological station in the city of Modena and are updated every hour. Then, some changes are introduced into the model framework (for example, an ad hoc defined mitigation measure) and RayMan is run under the changed settings (scenario runs): the aforementioned thermal indices are again computed and the difference in their values between a selected scenario and the reference run may be thus considered as the net effect of the mitigation measures on the thermal environment, and consequently, on the bio-meteorological conditions. In this respect, the use of meteorological conditions referring to a specific day is not essential, since the focus of the study is the impact of the introduction of some mitigation measures into the urban fabric.

One of the most relevant parameters characterizing the location where the computation of thermal indices is made is the so-called Sky View Factor (SVF): urban structures which fall within the model integration domain reduce the visibility of the reference human body located in the center of the domain itself (SVF $=1$ in case of no obstacle being present within the integration domain). Thus, SVF provides an estimation of the modification to the visible horizon, which in turn plays a crucial role in the modification of the radiation fluxes which enter the heat balance. For this reason, SVF values are reported nearby the reference run as well as the scenarios, in order to give an idea of the impact of obstacles surrounding the reference human body.

The reference run is the control situation against which the different scenarios are compared; the list of these scenarios is summarized as follows, with the indication of the values for surface albedo $\left(a_{s}\right)$ and for sky view factor (SVF):

- 'CONTROL' is the reference run for the current situation (Fig. 3 upper), with $a_{s}=0.15$ (to represent old asphalt) and SVF $=0.635$;

- '5M_CLIP'; '7M_CLIP', '9M_CLIP' are scenarios in which the domain is the same as 'CONTROL', but the building heights are clipped at 5 meters (SVF $=0.756$ ), 7 meters (SVF $=0.688$ ) and 9 meters $(S V F=0.653)$, respectively;

- 'GRASS' is the scenario in which the domain is the same as 'CONTROL', with the same sky view factor, but $a_{s}=0.30$ (to 
represent a grass surface instead of the old asphalt);

- 'CON_TREE', 'DEC_TREE' are the scenarios with the insertion of two lines of trees in a row at the edge of the car park (Fig. 3b); SVF $=0.570$ for CONifer trees and $S V F=0.507$ for DECiduous trees, the difference in SVF values between the two scenarios being due only to the tree crowns;

- 'GRASS_CON', 'GRASS_DEC' are the scenarios with a grass surface $\left(a_{s}=0.30\right)$ and two lines of different trees at the edge of the car park.

The thermal comfort indices (PET, UTCI) are computed in the center of the selected
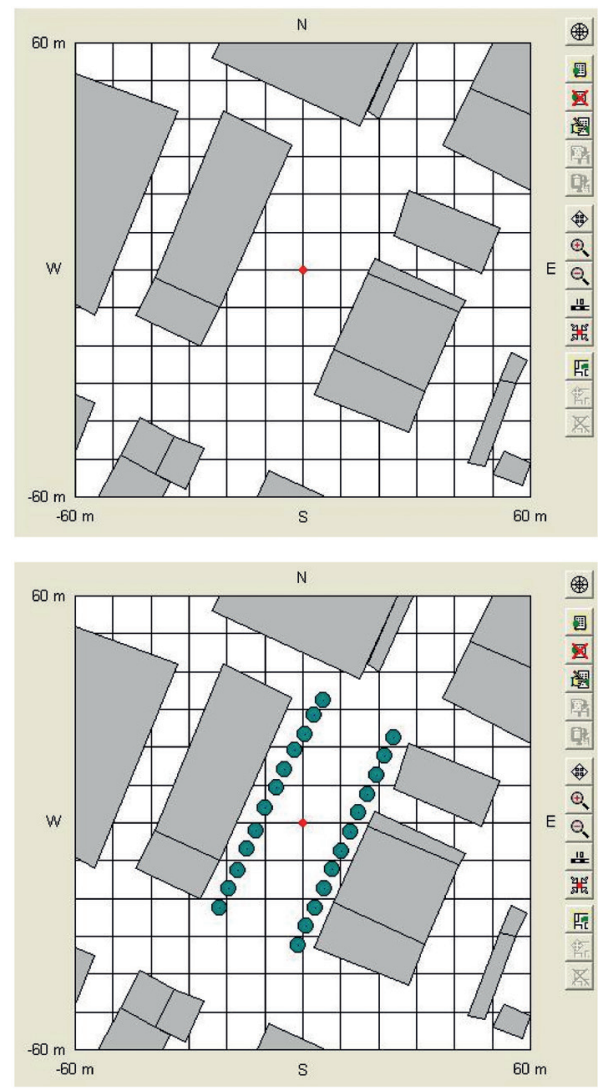

Figure 3. RayMan domain with: (upper) the actual obstacle distribution; (lower) the obstacle distribution with the insertion of two lines of trees (conifers and deciduous trees are in exactly the same position). The values of the thermal indices refer to the central point of the domain (in red) domain to represent bio-meteorological conditions caused by the urban settlements on the human being who is supposed to stand in that place.

The values of both indices, related to the thermal conditions, are considered in this paper specifically in order to assess the difference between the reference situation and the various scenarios, rather than the absolute value of the indices themselves. In fact, the analysis does not refer to a specific period (such as during an heat wave): to the contrary, RayMan meteorological input has been taken from the weather conditions of a typical August day in the city of Modena, a choice which is related to the focus on the improvement and/or worsening of bio-meteorological conditions associated with a specific mitigation measure rather than on more specific conditions, such as heat waves. For this reason, plots in the following section are shown in the form of bar-plots, where each bar-plot represents the difference between a scenario and the CONTROL during the course of the day, starting at 00:00 until 00:00 next day. Thus, it is possible to show how much and when the specific scenario either improves or worsens the hourly bioclimatic conditions. In general, positive values of the bar-plots indicate that the CONTROL run is cooler than the considered scenario, while negative values of the bar-plots indicate that the scenario run is cooler than CONTROL run.

\section{Results}

A preliminary analysis was performed in order to evaluate the sensitivity of the RayMan model to building height distribution. In this analysis, building heights were clipped to certain specific values: scenarios with maximum heights of 5,7 and 9 meters were considered and were compared to the results obtained in the CONTROL run; on the other hand, building locations and relative positions were left unaltered. The bar-plot differences between each scenario and the CONTROL run are shown in Figure 4. 
What clearly emerges from Figure 4 is that scenario runs with clipped heights are generally cooler than the CONTROL run, especially during night-time. In particular, the scenario with the lowest building (5M_CLIP) has the largest positive impact in terms of thermal comfort. On the other hand, the CONTROL run is relatively cooler in respect to each scenario for a couple of hours, the first after sunrise and the other before sunset, when the shadows projected by buildings which are taller than in each scenario certainly play a crucial role in the reduction of air temperature and, consequently, of bio-climatic discomfort conditions.

Other scenarios were defined with particular emphasis placed on the insertion of vege- length and the trunk diameter. The trees were inserted in two parallel lines in a row at the edges of the car park (see Fig. 3 lower); the remaining domain was left unaltered in terms of the material of pavements and so on.

All the scenarios concerning the presence of trees (conifer and deciduous trees) were run both at an early stage in tree life and when trees are already developed: the former scenarios are not presented in the following figures since the insertion of small trees is basically irrelevant with respect to the thermal conditions.

Figure 5 shows the differences during the course of the day between the CONTROL run on one side and scenarios with the insertion of two lines of trees in a row. The differences

\section{Universal Thermal Climate Index}

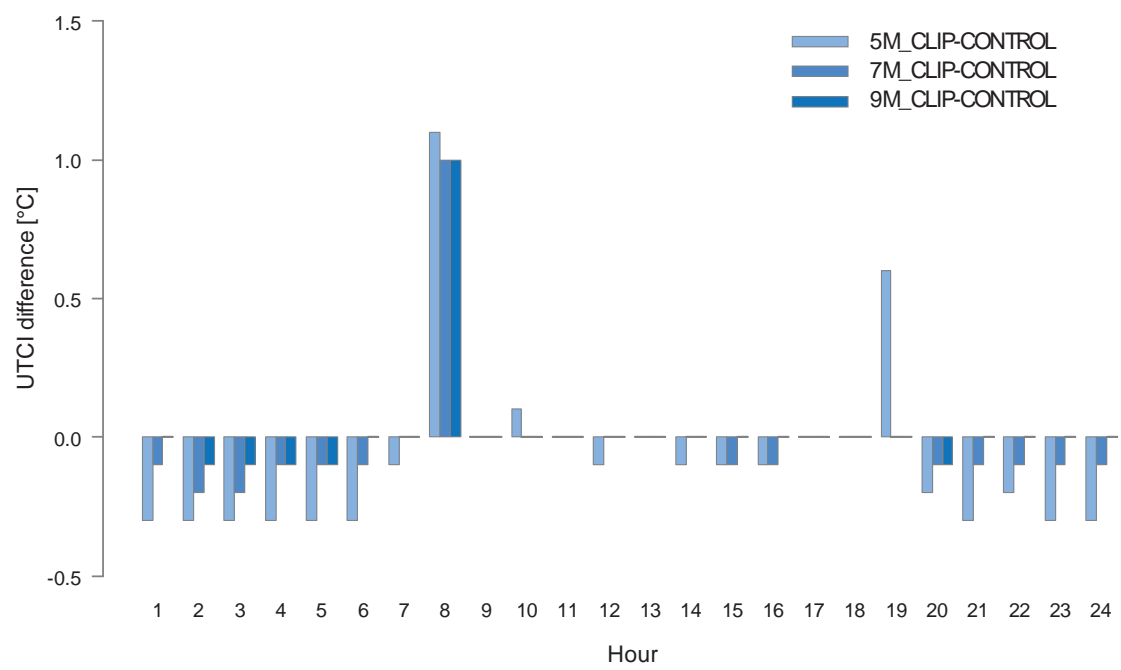

Figure 4. Bar-plots represent UTCl hourly difference $\left[{ }^{\circ} \mathrm{C}\right]$ during the course of the day (August 5th, 2012) between each scenario with modified building heights (see text for the explanation of scenario labels) and the CONTROL run

tation, such as trees (either conifers or deciduous trees) at different stages of development and/or grass on the ground surface. As for the first set of simulations, the RayMan model offers the possibility of shaping trees inside the domain at different stages of development, through setting 4 different parameters, i.e. the tree height, the crown radius, the trunk between the two scenarios depend on the kind of trees, conifers on one side (CON_ TREE) and deciduous trees on the other side (DEC_TREE): in this Summer situation, the difference between the two is certainly not related to the presence of leaves (which are present in both types of trees in this period of the year), but rather to the shape of the 


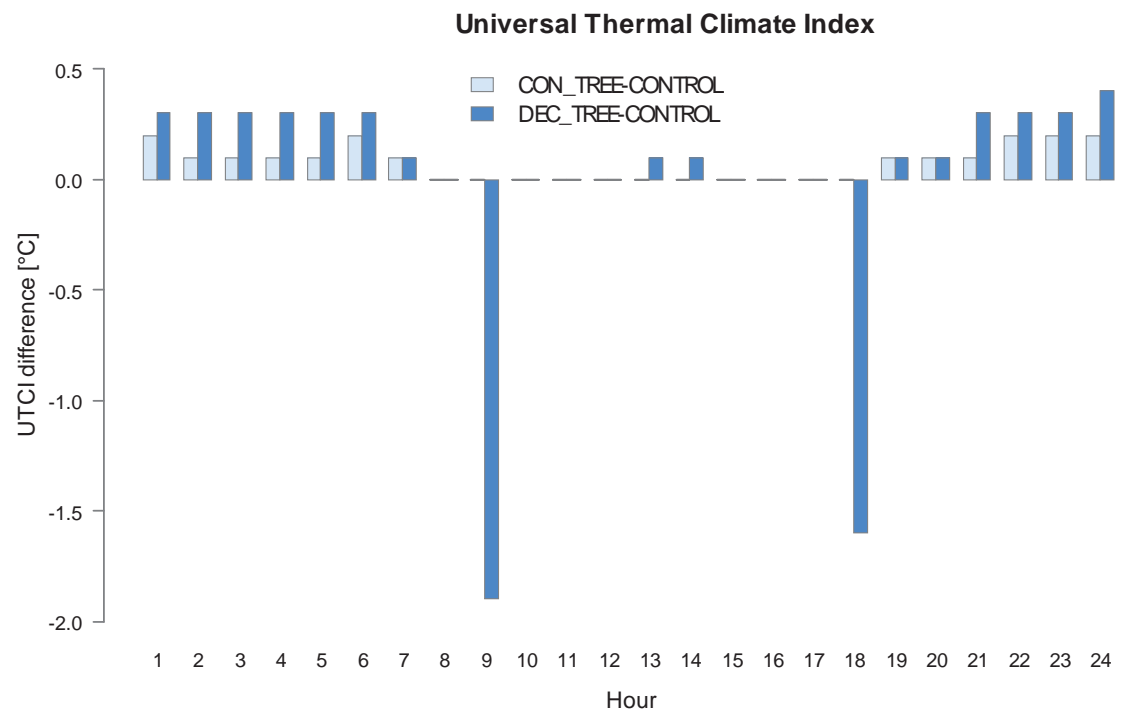

Figure 5. Bar-plots represent UTCl hourly difference $\left[{ }^{\circ} \mathrm{C}\right]$ during the course of the day (August 5 th, 2012) between each scenario with the insertion of trees (see text for the explanation of scenario labels) and the CONTROL run

tree crown as represented in the RayMan model: this representation has a rather different impact in terms of the projected shadow, as could be shown by the couple of hours after sunrise and before sunset when the larger crowns of deciduous trees are quite effective, while that of conifers are not. On the other hand, during night-time, the presence of trees is accompanied by a certain worsening of thermal comfort with respect to the CONTROL run, probably because of the current representation of trees' biological mechanisms, such as an increase in water vapor content released into the atmosphere.

Figure 6 shows the results obtained with the insertion of grass at the ground surface of the car park, instead of asphalt, which is an impervious material (GRASS); in addition, this scenario was also enriched by the addition of two lines of trees, as previously described (GRASS_CON and GRASS_DEC). The GRASS scenario shows a certain, beneficial effect on diurnal thermal comfort, as can be expected during day-time, when the grass surface (with albedo lower than the asphalt surface) reflects less short-wave radiation to the atmosphere. Therefore UTCl values are generally smaller during day time, while during the night, the radiative properties of the pervious grass surface replacing the old asphalt has no substantial effect.

The insertion of the two lines of trees together with grass at the ground surface (GRASS_CON and GRASS_DEC) is again more relevant during night-time, as also emerges from Figure 5; during day-time it appears to have almost no effect: in general the reduction of the thermal discomfort in these scenarios seems almost completely due to the presence of grass, except for the couple of hours after sunrise and before sunset when sunlight is blocked by the presence of trees (in particular by deciduous trees) which are represented in the RayMan model with larger crowns so that the projected shadow shows a very relevant effect on the reduction of bio-climatic discomfort. 


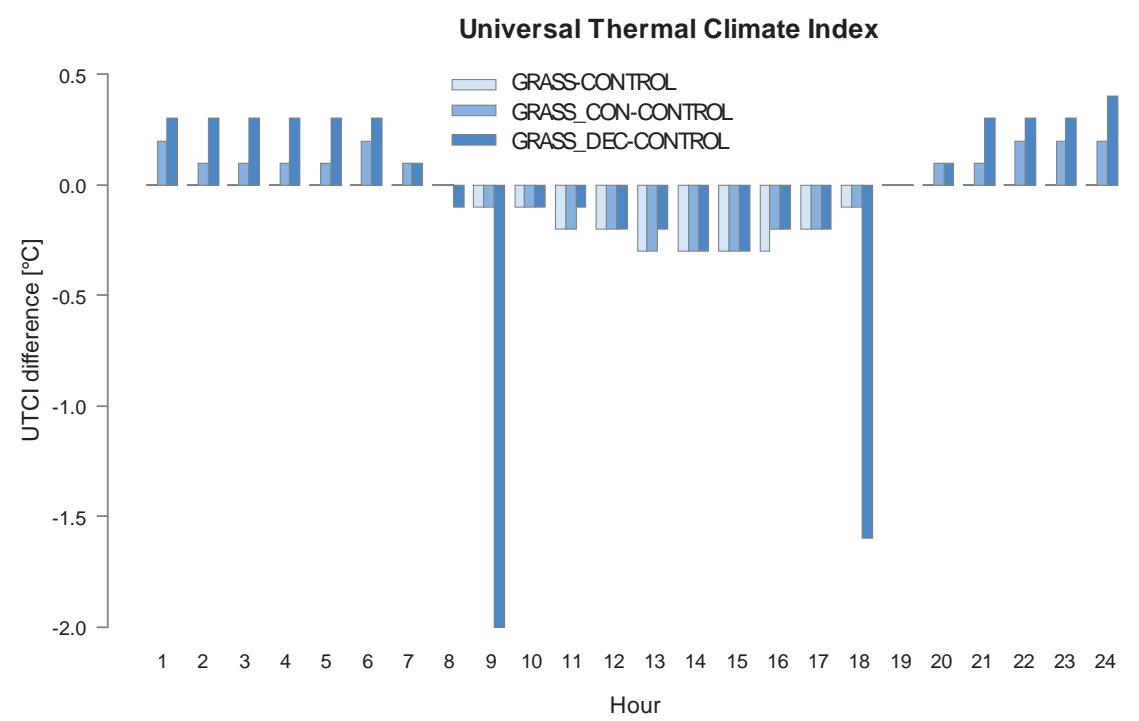

Figure 6. Bar-plots represent UTCl hourly difference $\left[{ }^{\circ} \mathrm{C}\right]$ during the course of the day (August 5 th, 2012) between each scenario with the insertion of a grass surface instead of asphalt with or without the additional insertion of trees (see text for the explanation of scenario labels) and the CONTROL run

\section{Discussion}

The point of view of the previous analysis is certainly partial, in the sense that it was not intended to estimate the overall impact of certain mitigation measures of the urban heat island effect, in terms of a decrease in atmospheric temperature. Rather, it should be considered as an attempt to estimate the effects on a human being in terms of thermal comfort in a certain urban background and in the presence of some specific mitigation measures. From this point of view some remarks can be pointed out.

The focal point is that the effective mitigation measures which were considered in the previous section of the paper are mainly related to the insertion of green zones, a measure which is not particularly burdensome on the urban fabric, since it is not related to the need to modify specific characteristics of buildings. The municipality of Modena does not foresee any public, overall intervention inside Villaggio Artigiano: only projects developed by the owners of the different parcels in the area will be taken into consideration. In case of approval, interventions will ultimately be co-financed by the Municipality, according to some specific rules, which are currently under discussion in the framework of urban redevelopment.

From the point of view of thermal comfort, it is quite clear that the most effective discomfort reduction is obtained by introducing trees into the terrain: the projected shadows from the trees produce the largest reduction of bioclimatic discomfort as is clearly shown in Figures 5 and 6 in the hours after sunrise and before sunset: the mitigation effect can be estimated at an hourly reduction of about 2 degrees in the scale of the thermal index; apart from this couple of hours when the shading effect is present, trees are more or less irrelevant during day-time in this configuration.

These results, obtained in presence of trees, certainly require further investigation, also because of the almost dichotomous effect on the thermal indices due to the projected shadow, with very sharp peaks in some hours and almost no impact in the very next hours. 
As a further remark, the replacement of the asphalt and/or concrete pavement with a pervious surface (grass) has a positive impact on the thermal comfort; it must be underlined that the absolute value of this effect is much less relevant than tree shadows: in fact, the maximum hourly differences amount to $0.2-0.3^{\circ} \mathrm{C}$. Nevertheless, the effect is present in the whole period between sunrise and sunset and it progressively increases, reaching its maximum during the central hours of the day, when maximum air temperature is reached.

The results obtained with the modification of building heights show rather small differences in the values of bio-climatic indices: this emphasizes the importance of the presence of green areas, which is the most efficient way to reduce the urban heat island effect. This can be considered as very important information, since this kind of intervention is certainly less expensive, with respect to those related to modifications of the existing buildings.

The effective involvement of local stakeholders from the Municipality of Modena as well as from the Emilia-Romagna Region in the UHI-project, together with the close collaboration of scientific and administrative partners on a local scale, is of fundamental importance in order to be aware of the results of the analysis and to take it into proper consideration within common urban planning tools.

\section{Acknowledgements}

This work is financially supported by the transnational cooperation project within the Central Europe Programme 'Development and application of mitigation and adaptation strategies and measures for counteracting the global Urban Heat Islands phenomenon'. This project is implemented through the CENTRAL Europe Programme co-financed by the ERDF.

\section{Editors' note:}

Unless otherwise stated, the sources of tables and figures are the author(s), on the basis of their own research.

\section{References}

BŁAŻEJCZYK K., JendRITZKY G., BRÖDE P., FIAla D., Havenith G., Epstein Y., Psikuta A., KampMANN B., 2013. An introduction to the Universal Thermal Climate Index (UTCI). Geographia Polonica, vol. 86, no. 1, pp. 5-10.

Büttner K., 1938. Physikalische Bioklimatologie. Leipzig: Akademische Verlagsgesellschaft.

Fanger P.O., 1970. Thermal comfort: Analysis and applications in environmental engineering. Copenhagen: Danish Technical Press.

HÖPPE P., 1999. The physiological equivalent temperature - a universal index for the biometeorological assessment of the thermal environment. International Journal of Biometeorology, vol. 43, no. 2, pp. $71-75$.

Matzarakis A., Matushek O., 2011. Sky view factor as a parameter in applied climatology - rapid estimation by the SkyHelios model. Meteorologische Zeitschrift, vol. 20, no. 1, pp. 39-45.

Matzarakis A., Mayer H., IzIomon M.G., 1999. Applications of a universal thermal index: physiological equivalent temperature. International Journal of Biometeorology, vol. 43, no. 2, pp. 76-84.

Matzarakis A., Rutz F., Mayer H., 2007. Modelling radiation fluxes in simple and complex environments - application of the RayMan model. International Journal of Biometeorology, vol. 51, no. 4, pp. 323-334.

Matzarakis A., Rutz F., Mayer H., 2010. Modelling radiation fluxes in simple and complex environments: basics of the RayMan model. International Journal of Biometeorology, vol. 54, no. 2, pp. 131-139. 